\title{
Diver Observations by Means of Acoustic Methods
}

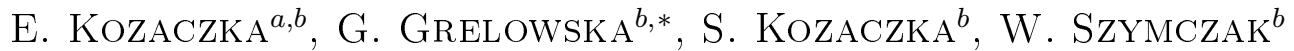 \\ ${ }^{a}$ Gdansk University of Technology, Narutowicza 11/12, 80-233 Gdańsk, Poland \\ ${ }^{b}$ Polish Naval Academy, Smidowicza 69, 81-103 Gdynia, Poland
}

\begin{abstract}
Searching for objects, especially small ones, moving under water near its the free surface, is always not an easy task. Designing tools for the detection of such targets is a real challenge when the possibility of a terrorist attack is a real threat. This paper presents some aspects of diver detection by means of acoustics methods, both active (side scan sonar) and passive ones (linear receiving antenna). This approach is quite effective because divers can use both breathing apparatus operating in the closed-loop (so-called rebreathers), and popular open-circuit breathing (exhalation of used air or a breathing gas mixture into the water).
\end{abstract}

DOI: $10.12693 /$ APhysPolA.123.1098

PACS: 43.30.Vh, 43.30.Wi, 43.66.Qp

\section{Introduction}

Detection of objects that are living organisms with respiratory system suitable for breathing in a gaseous environment during their activity in water is a specific task [1]. They must have a device that supplies air or a breathing gas mixture in order to stay under water for an extended period of time. Classical units to supply the human respiratory system consist of a cylinder containing compressed air or a breathing gas mixture and a pressure reduction unit. The most common are two coupled reduction systems adjusting pressure to the respiratory system.

Commonly used breathing apparatus for diving works in the following way. Compressed breathing means is adjusted to the pressure adapted to the human respiratory organs at given depth. Air exhaled directly into water creates a cloud of gas bubbles that are the source of acoustic waves and also the perfect reflector for the incident acoustic waves, due to the very large difference of acoustic impedance of air and water. The detection range for a diver will depend strongly on the target strength and very little data are available in the literature on this topic [2].

Security of marine infrastructure such as bridges, ports, harbor facilities, etc. is of increased importance [3]. A threat to these facilities can come from scuba divers and acoustic methods for detection of such divers are being investigated.

Acoustic methods have been extensively used to locate and identify underwater objects [4]. These applications include locating ships and underwater vehicles [5-7], finding shipwrecks [8], imaging sediments [9] and imaging bubble fields. Acoustics is the most effective tool for monitoring this environment because the sound has the ability to propagate over long distance in water [10].

*corresponding author; e-mail: g.grelowska@amw.gdynia.pl
The main unknown in assessing the possibility to use a sonar to detect divers is their target strength.

In this case, two methods can be used for detecting this kind of target. One of them uses passive observation methods by means of a linear or spatial antenna composed of detectors (hydrophones) that allow tracking a diver. This is a relatively universal method having additional advantage that the object is tracked without its knowledge.

The other type of observation is the use of active methods involving the acoustic scanning of the observed area. In this case, the secondary source of the wave incident on the diver will be floating gas (air) bubbles as well as pressurized gas cylinders. In addition, the diver's body is a good object reflecting sound waves, in particular when a diver wears a diving suit called the dry one, and it is the rule in the case of cold water.

In the case of systems with closed circuit breathing where there is no effect of gas bubbles, the secondary sources (parts reflecting sound waves) are mainly gas cylinders, breathing circuit, and the body of the diver. In such a situation, active methods are more effective, with particular emphasis on the waves reflected from the axially symmetric containers such as gas cylinders, both single and double.

In the following part of the paper, results of measurements for all the aforementioned cases will be presented.

\section{Target strength of diver}

Target strength is one of the most important parameters of the object in active method of underwater observation. The diver is more complicated object than a finite cylinder due to the equipment like tanks, suit etc. that also contributes strongly to the total scattering. But at first step to assess roughly the target strength of the diver we used in modeling the assumption that the main component of such object is the gas container that could be treated as a finite cylinder [11]. The solution is most accurate in the case where the incident plane wave is normal to the axis of the cylinder and the length of the cylinder is greater than the diameter (Fig. 1). 


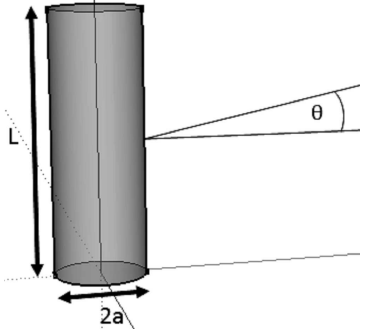

Fig. 1. Cylinder of length $L$, radius $a$ and the ray incident at angle $\theta$.

The target strength was calculated with the use of the numerical modeling of a finite cylinder using the formula from Urick [12]:

$$
T S=10 \log \frac{a L^{2}}{2 \lambda}\left[\frac{\sin (k L \sin \theta)}{k L \sin \theta}\right]^{2} \cos \theta
$$

where $L$ is the cylinder length $[\mathrm{m}], a$ is the cylinder radius $[\mathrm{m}], \theta$ is the wave incident angle, and $k=2 \pi / \lambda$ is the wave number.

The target strength calculated with the use of the numerical modeling of a finite cylinder of length $1 \mathrm{~m}$ and radius $0.125 \mathrm{~m}$ using the formula (1) is shown in Fig. 2. The figure also shows results of calculations done for the cylinder that could be used as a model of a pipeline of $0.1 \mathrm{~m}$ radius when is observed with the use of wave with frequency of $15 \mathrm{kHz}$ (typical for parametric echosounder) as well as $450 \mathrm{kHz}$ used in the side scan sonar.

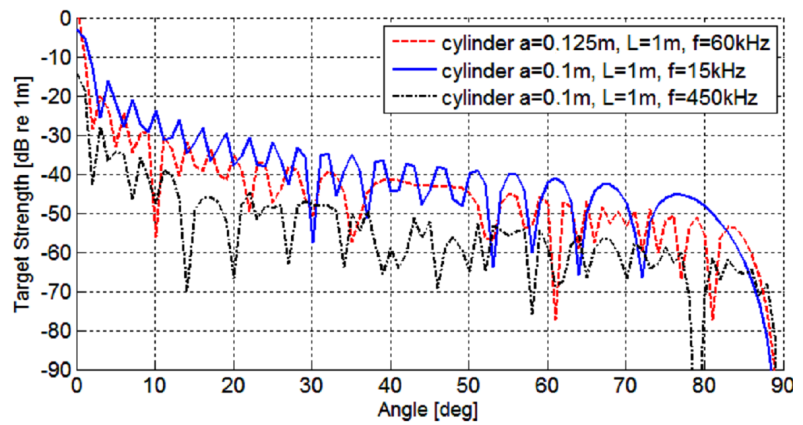

Fig. 2. Results of target strength calculation for cylinder modeling the diving tank and the pipeline for different frequencies of waves.

In consecutive figures, echograms pointed out characteristic elements in shapes of underwater image of a diver are shown. The echogram in Fig. 3 was taken by means of side scan sonar during tests in the area of the Gulf of Gdansk.

Another type of sonar gives an image of a diver slightly different from the one shown in Fig. 3.

In Figs. 4 and 5 there is a well visible silhouette of a diver with many details. Further, Fig. 6 shows the echogram of underwater pipeline taken by means of parametric echosounder SES 2000 whose target strength estimation is given in Fig. $2-$ solid line, cylinder $a=0.1 \mathrm{~m}$, $L=1 \mathrm{~m}, f=15 \mathrm{kHz}$.

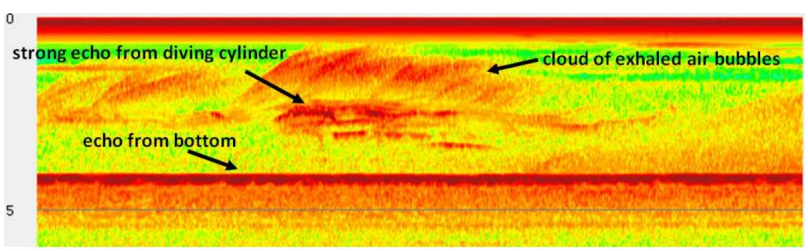

Fig. 3. Echogram of a diver close to the bottom taken by side scan sonar.

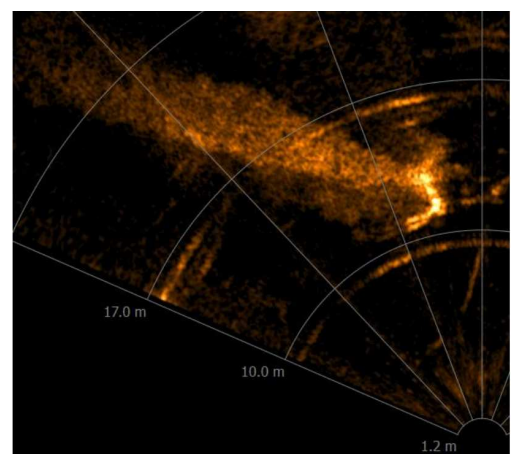

Fig. 4. Echogram of diver taken by multibeam sonar BlueView P450.

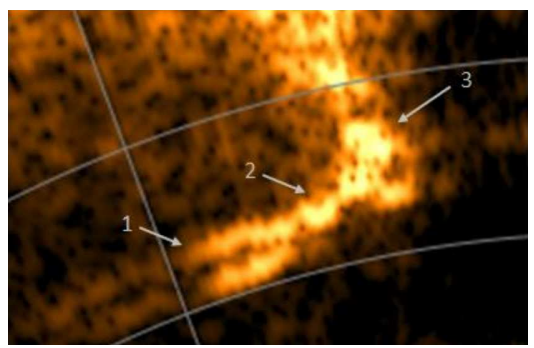

Fig. 5. Details in underwater silhouette of diver in echogram from Fig. 4: 1 - diver's legs, 2 - diving cylinder, 3 - cloud of bubbles near breathing machine.

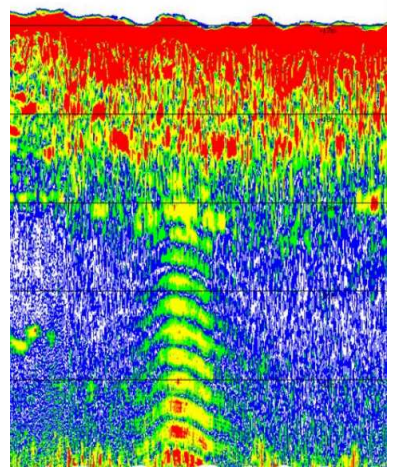

Fig. 6. The echogram of the pipeline taken by parametric echosounder SES2000. 


\section{Passive method in diver observation}

Passive method in underwater observation is especially useful in protection system for objects of particular importance such as ports, farwaters, oil rigs, and others. In such a system the most important thing is to notice changes in the ambient noise. The distortion of the ambient noise produced by a diver is very small when taking into account the difference in the pressure value. Therefore characteristics of sound of a diver in time as well as in the frequency domain should be considered.

The first step towards assessing the sound produced by a diver breathing with open circuit machine was taking the characteristics of breath when the diver was close to the hydrophone. The recorded signal of pressure and the pressure level determined based on it is shown in Fig. 7 .

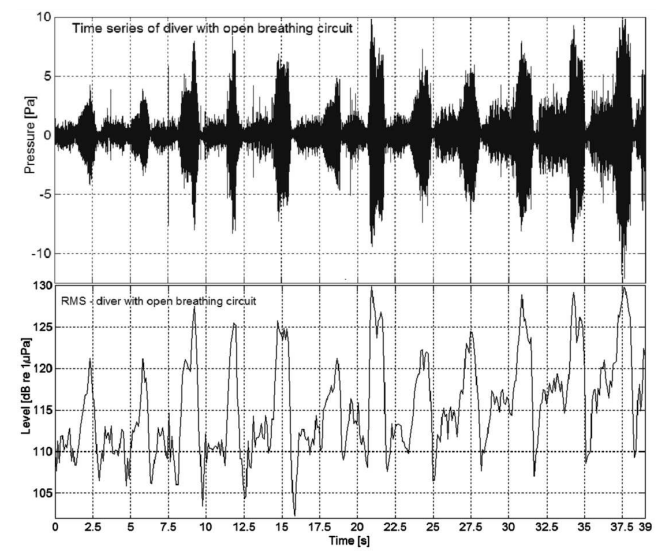

Fig. 7. Changes in pressure (upper panel) and in pressure level (lower panel) in sound produced by a diver.

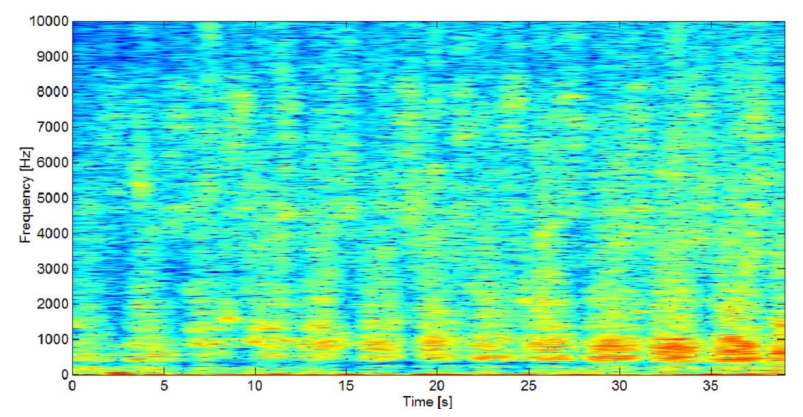

Fig. 8. Spectrogram of a diver using an open breathing circuit.

The main changes that can be noticed in the time series chart are caused by exhausted air in the form of bubbles rapidly changing their volume while floating upwards. Sound that can be heard during inhale is recognizable but not so well distinguished as this related to exhaust. The level of underwater noise varies in the range of about 10-15 dB.

In real conditions where the diver is in certain distance from a sensor, the results of spectral analysis could be more useful. An example of spectrogram of diver's sound is given in Fig. 8. Except from variation in pressure as a function of time, the presence of a diver is a source of consecutive changes in spectrum of environmental noise in range of frequency up to $10 \mathrm{kHz}$, whereas the greatest changes are observed in band up to $2 \mathrm{kHz}$. In this example a diver was moving slowly in the direction of the hydrophone array.

\section{Conclusions}

Detection of a human diver by means of passive method is rather a complicated task because the object represents a broadband source of small intensity. Determining the presence of the diver needs a discerning analysis of the environmental noise simultaneously in time and frequency range.

For active sonars, the diver is quite a well recognizable object. The diving cylinder, the dry diving suite, and the cloud of bubbles when the open circuit is in use create highly reflective elements.

\section{Acknowledgments}

The investigation was partially supported by the National Center for Research and Development, Grant No O R00 008909 and Grant No O ROB 0007 01/ID7/1.

\section{References}

[1] D.B. Reeder, T.K. Stanton, J. Acoust. Soc. Am. 116, 729 (2004).

[2] S. Sarangapani, J.H. Miller, G.R. Potty, D.B. Reeder, T.K. Stanton, D. Chu, IEEE Conference Publications, Oceans 2005 - Europe, 2, 1010 (2005).

[3] M Kastek, R. Dulski, M. Zyczkowski, M. Szustakowski, P. Trzaskawka, W. Ciurapinski, G. Grelowska, I. Gloza, S. Milewski, K. Listewnik, Proc. SPIE, Unattended Ground, Sea, and Air Sensor Technologies and Applications 8388, 83880M (2012).

[4] E. Kozaczka, J. Domagalski, I. Gloza, Polish Maritime Res. 17(3), 26 (2010).

[5] E. Kozaczka, Archiv. Acoust. 13, 121 (1978).

[6] E. Kozaczka, Archiv. Acoust. 14, 221 (1979).

[7] M. Iwaniec, J. Wiciak, Acta Acust. \& Acust. 88, 722 (2002).

[8] E. Kozaczka, G. Grelowska, W. Szymczak, S. Kozaczka, Polish Maritime Research 19,(4)3 (2012).

[9] G. Grelowska, E. Kozaczka, Acta Phys. Pol. A 118, 66 (2010).

[10] G. Grelowska, Archiv. Acoust. 25, 359 (2000).

[11] T.K. Stanton, P.H. Wiebe, D. Chu, J. Acoust. Soc. Am. 103, 254 (1997).

[12] R. J. Urick, Principles of Underwater Sound, Ch. 10, Mc Graw-Hill, New York 1975. 